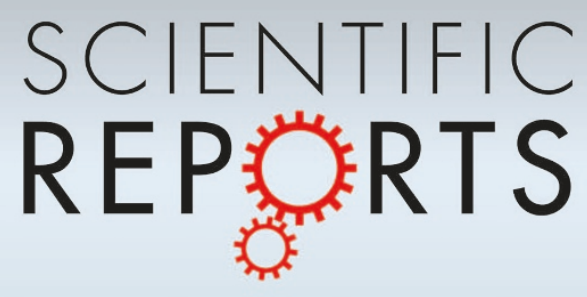

OPEN

SUBJECT AREAS:

FOCAL ADHESION

CELLULAR NEUROSCIENCE

Received

20 October 2014

Accepted

29 December 2014

Published

22 January 2015

Correspondence and requests for materials should be addressed to

J.L. (jialuo888@uky. edu) or Z.J.K.

(kezunji@shutcm.edu.

$\mathrm{cn})$

* These authors contributed equally to this work.

\section{PACT/RAX Regulates the Migration of Cerebellar Granule Neurons in the Developing Cerebellum}

Yue Yong 1,3*, Ya Meng 1,3*, Hanqing Ding' ' Zhiqin Fan ${ }^{3}$, YifenTang ${ }^{3}$, Chenghua Zhou 1,3, Jia Luo 2 \& Zun-Ji Ke $\mathrm{Ke}^{1,3}$

'Department of Biochemistry, Shanghai University of Traditional Chinese Medicine, 1200 Cailun Road, Shanghai 201203, China, ${ }^{2}$ Department of Pharmacology and Nutritonal Sciences, University of Kentucky College of Medicine, Lexington, Kentucky 40536, U.S.A, ${ }^{3}$ Key Laboratory of Nutrition and Metabolism, Institute for Nutritional Sciences, Shanghai Institutes for Biological Sciences, Chinese Academy of Sciences, Graduate School of the Chinese Academy of Sciences, Shanghai 200031, China.

PACT and its murine ortholog RAX were originally identified as a protein activator for the dsRNA-dependent, interferon-inducible protein kinase PKR. Recent studies indicated that RAX played a role in embryogenesis and neuronal development. In this study, we investigated the expression of RAX during the postnatal development of the mouse cerebellum and its role in the migration of cerebellar granule neurons (CGNs). High expression of RAX was observed in the cerebellum from postnatal day (PD) 4 to PD9, a period when the CGNs migrate from the external granule layer (EGL) to the internal granule layer (IGL). The migration of the EGL progenitor cells in vivo was inhibited by RAX knockdown on PD4. This finding was confirmed by in vitro studies showing that RAX knockdown impaired the migration of CGNs in cerebellar microexplants. PACT/RAX-regulated migration required its third motif and was independent of PKR. PACT/RAX interacted with focal adhesion kinase (FAK) and PACT/RAX knockdown disturbed the FAK phosphorylation in CGNs. These findings demonstrated a novel function of PACT/RAX in the regulation of neuronal migration.

$\mathrm{P}$ rotein kinase, interferon-inducible double stranded RNA dependent activator (PACT) and its murine ortholog RAX were independently discovered as the protein activator for the double strand RNA (dsRNA)-dependent, interferon-inducible protein kinase (PKR $)^{1,2}$. PACT and RAX are almost identical in their amino acid sequences and they belong to an evolutionarily conserved family of RNA-binding proteins ${ }^{3}$. Under various stress conditions ${ }^{4-8}$, PACT/RAX binds to PKR through its two dsRNA binding motifs (dsRBMs), and regulates the conformational change of PKR through its third motif, resulting in PKR autophosphorylation ${ }^{9}$ and then the phosphorylation of eukaryotic initiation factor $2 \alpha$ (eIF $2 \alpha$ ), leading to the inhibition of protein synthesis and the induction of apoptosis ${ }^{10}$. PACT also interacts with Dicer to facilitate the maturing process of small RNAs ${ }^{11,12}$. The depletion of PACT affects the accumulation of mature microRNAs (miRNAs) and reduces the efficiency of small interfering RNA (siRNA)-induced RNA interference (RNAi) ${ }^{13}$.

The ablation of the $8^{\text {th }}$ exon in the prkra gene in mice induces severe microtia, impaired hearing, reduced body size and fertility defects ${ }^{14,15}$. Missense mutation in the second dsRBM of the prkra gene causes deficits in growth, ear development, craniofacial development and ovarian structure ${ }^{16}$. In addition, deletion of the entire RAX gene is embryonic lethal in mice at the pre-implantation stage. In fruit flies, a transposon insertion in the $5^{\prime}$-UTR of dRax (independently identified as loqs/R3D1) induces a highly abnormal commissural axon structure of the central nervous system (CNS) and 70\% of the flies homozygous for the mutant allele die prior to adulthood ${ }^{17}$. All these findings suggest that PACT/RAX plays an important role in embryogenesis and development.

Focal adhesion kinase (FAK) is a tyrosine kinase localizing at the focal adhesions ${ }^{18}$. The regulatory role of FAK or paxillin in cell migration has been well established ${ }^{18,19}$. In neurons, phosphorylation of FAK at serine 732 is critical for the organization of a small network of microtubules that partially encompass the nucleus, which is important for neuronal migration ${ }^{20}$. Mice with neuron/glia-specific FAK ablation show impaired cerebellar foliation, such as variable decreases in foliation sizes and the lack of intercrural and precentral fissures ${ }^{21}$.

In this study, we show that the expression of RAX in the cerebellum is developmentally regulated. RAX knockdown impairs cerebellar granule neuron (CGN) migration. The third conserved motif of PACT/RAX is 
required for its role in migration which is independent of PKR and may be mediated by its interaction with FAK. These results reveal a novel role of PACT/RAX in regulating neuronal migration during the development.

\section{Results}

Developmental expression of RAX in mouse cerebellum. To explore the role of PACT/RAX in cerebellar development, we first examined the developmental expression of RAX in mouse cerebellum. High level of RAX was observed in the cerebellum on PD4 and PD9; the expression decreased thereafter (Figure 1A). Compared to PD4, the expression of RAX decreased $70 \%, 86 \%$ and $94 \%$ by PD15, PD21, and adult, respectively (Figure 1B). The immunohistochemical (IHC) staining showed that RAX was highly expressed in EGL and Purkinje cell layer (PL) on PD4 and PD9 (Figure 1C), but the RAX-positive cells were only observed in Purkinje cells and interneurons in the internal granule layer (IGL) and molecular layer (ML) at PD15, PD21 and adulthood (Figure 1C). Confocal microscope images showed that RAX was expresseed in almost all cells in the EGL of PD4 mouse cerebellum (Supplementary Figure 1).

Role of RAX in the migration of cerebellar granule neurons (CGNs) in vivo. To explore the function of RAX in the developing cerebellum, the small interfering RNA (siRNA) constructs targeting RAX were delivered to the cerebellar granule precursors in the EGL of PD4 mice by in vivo electroporation. Enhanced yellow fluorescent protein (EYFP) was used as a marker of gene delivery. The RAX siRNA effectively down-regulated the expression of RAX in primary cerebellar granule neurons (CGNs) as determined by immunoblotting and immunofluorescence (Figures $2 \mathrm{~A}$ and $\mathrm{B}$ ). At $48 \mathrm{~h}$ post electroporation, some EYFP positive cells were observed migrating from EGL to IGL in the group transfected with a control siRNA; while few EYFP positive cells is migrating in the group transfected with RAX siRNA (Figure 2C). In the control group, $49 \%$ of the EYFPpositive cells migrated from outer EGL through inner EGL, and traveled through the ML to reach the IGL within 72 hours following the electroporation. However, only $23 \%$ of EYFP-positive cells migrated into IGL (Figures $2 \mathrm{C}$ and D) in mice treated with RAX siRNA; the migration from the outer EGL to the inner EGL seemed not affected by RAX knockdown (Figures 2C and D). Also, the migration depth of CGNs was greatly reduced in RAX knockdown mice as compared to control mice; it decreased from $105 \mu \mathrm{m}$ in control group to $58 \mu \mathrm{m}$ in RAX knockdown group at 72 hours after the electroporation (Figure 2E). At 96 hours after the electroporation, more EYFP-positive cells migrated to the IGL; but still fewer migrated cells were observed in mice treated with RAX siRNA (Figure 2C). The EYFP-positive cells in the IGL of control mice showed a typical post-migration morphology with multipolar dendrites (Figure 2F, arrow), while the EYFP-positive cells had a migrating bipolar morphology in the RAX knockdown mice (Figure 2F, double arrows) 96 hours post the electroporation. At 7 days post electroporation, $93 \%$ of the EYFP-positive cells migrated into IGL in the control group, while only 77\% of EYFP-positive cells were in IGL in the siRAX group (Supplementary Figure 2).

Bergman glia is the migration scaffold for CGNs and displayed no morphological difference in both control and RAX knockdown groups as revealed by GFAP staining (Figure 3A). RAX knockdown did not change cell proliferation since the percentage of ki67 positive cells showed no difference between the control siRNA- and RAX siRNA-treated groups (Figures 3B and C). At 7 days post electroporation, the EYFP-positive CGNs migrated into IGL in both groups differentiated and expressed NeuN (Figure 3D). We also examined the expression of cleaved caspase- 3 and show no difference between control and RAX knockdown groups (data not shown). Taken together, these results suggested that RAX knockdown inhibited the migration of CGNs without affecting the Bergman glia fibers, CGN proliferation/survival and post-migration differentiation.

RAX knockdown inhibits the migration of CGNs in cerebellar microexplants. We further examined the role of RAX in CGN migration by using the cultured cerebellar microexplants from

A

B
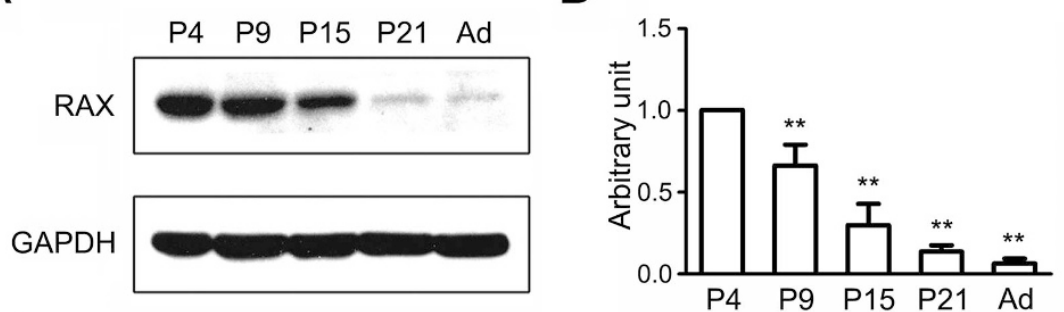

C

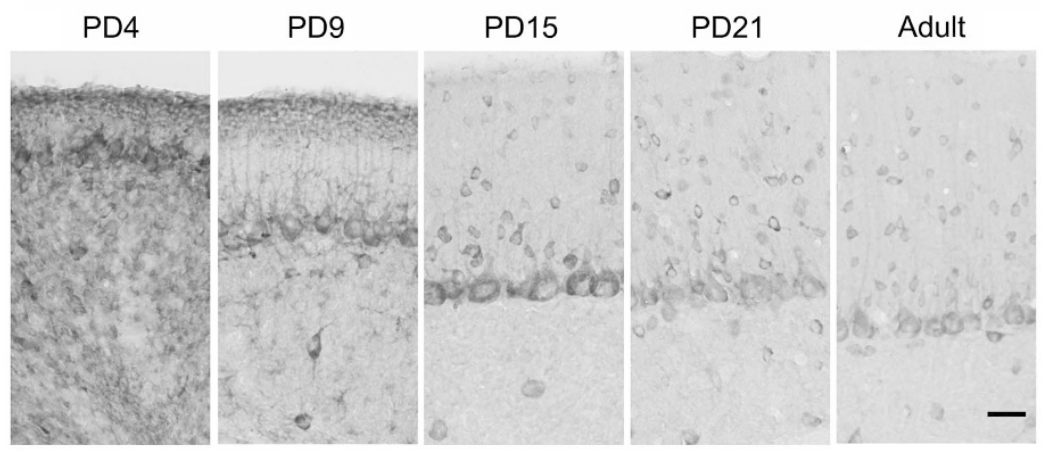

Figure 1 RAX expression in developing mouse cerebellum. (A) The expression of RAX protein in mouse cerebellum at PD4, PD9, PD15, PD21 and adult was measured by immunoblotting. The cropped lines are used and full-length immunoblots are shown in Supplementary Information section (Supplementary Figure 3A). (B) The expression of RAX was quantified and normalized to the loading control GAPDH. Each data point was mean \pm s.d. $(\mathrm{n}=3),{ }^{*} \mathrm{p}<0.01$. (C) The expression of RAX in the developing and adult mouse cerebellum was examined by immunohistochemistry (IHC). Scale bar $=20 \mu \mathrm{m}$. 
A

B

RAX

RAX/YFP
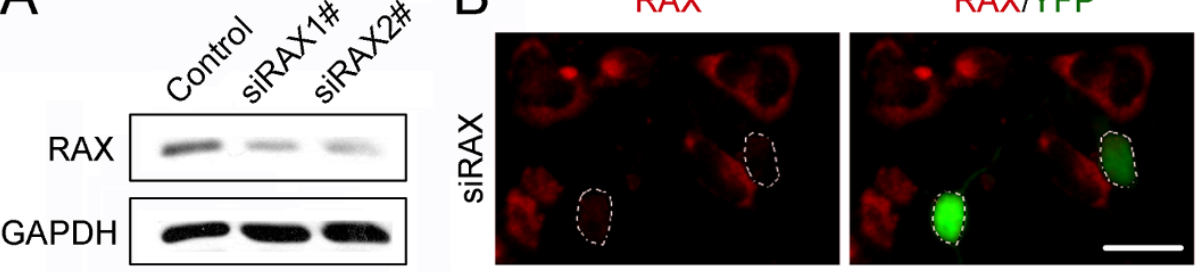

C

$48 h$

$72 \mathrm{~h}$

$96 \mathrm{~h}$
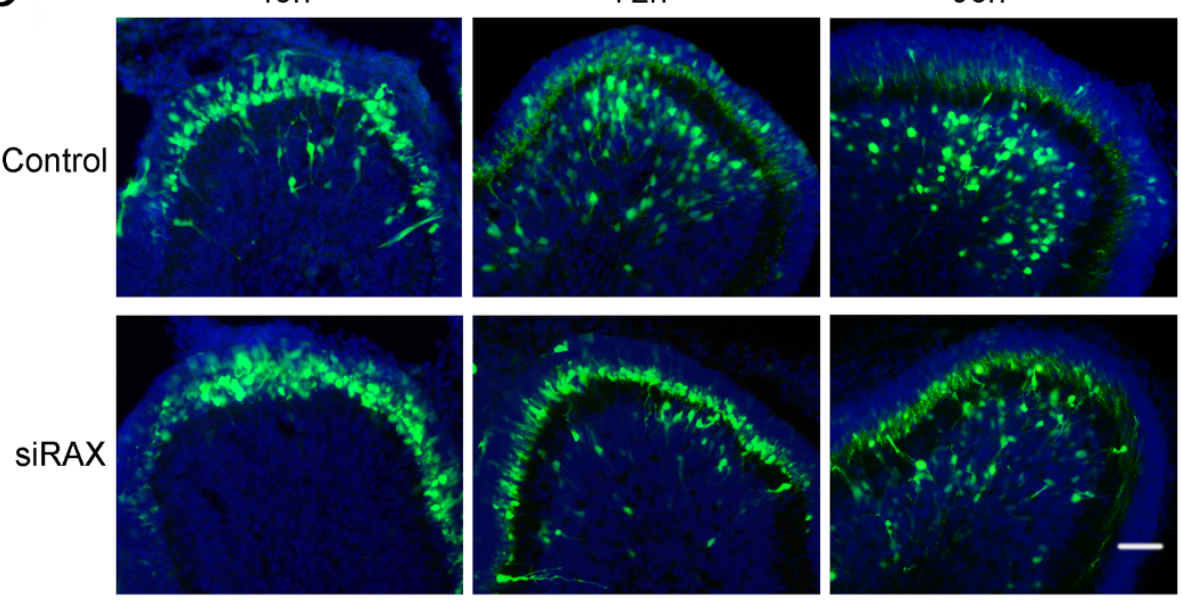

D

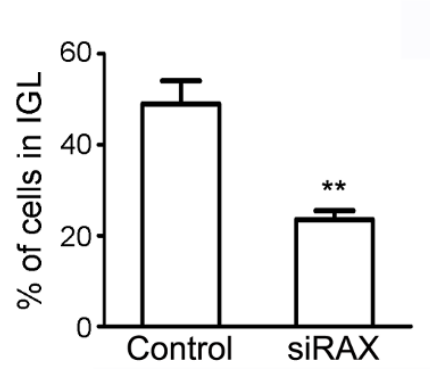

E

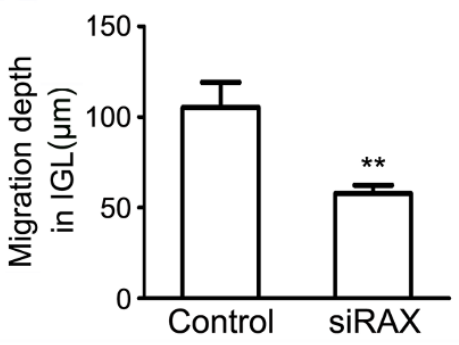

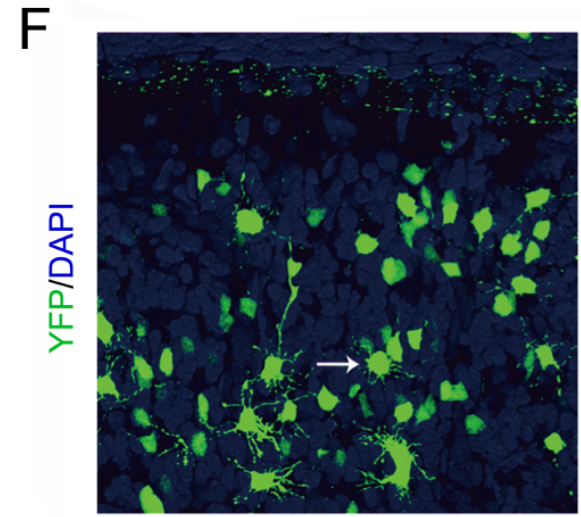

Control

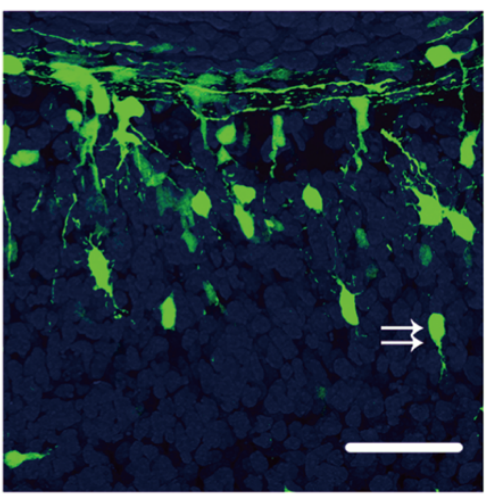

SiRAX

Figure 2 Effect of RAX knockdown on the migration of cerebellar granule neurons (CGNs). (A) Down-regulation of RAX after transfection of RAX siRNA (\#1 and \#2) for 48 hours was verified by immunoblotting in primary cultured CGNs. The cropped lines are used and full-length immunoblots are shown in Supplementary Information section (Supplementary Figure 3B). (B) Immunofluorescence images showing the RAX (red) expression in CGNs transfected with RAX siRNA for 48 hours (green) or untransfected. Scale bar $=20 \mu \mathrm{m}$. (C) siRNA for RAX and control siRNA were delivered to the cerebellum by in vivo electroporation at PD4. At 48, 72 or 96 hours post the electroporation, the mice were sacrificed and the sagittal sections of cerebella were examined under a fluorescent microscope as described under the Materials and Methods. Scale bar $=50 \mu \mathrm{m}$. (D) The percentage of cells that migrated into IGL was calculated at 72 hours post the electroporation. (E) The depth of cell migration into IGL at 72 hours post the electroporation. Each data point was mean \pm s.d. $(\mathrm{n} \geq 5)$, ** $\mathrm{p}<0.01$. (F) Coronal sections of cerebella showing CGNs in control and RAX siRNA (siRAX) treated groups 96 hours post the electroporation. CGNs in the IGL displayed post-migratory morphology of multi-polar processes (arrows in control group). Migrating CGNs showed tangential or radial migratory morphology of bipolar processes (double arrows in siRAX group). Scale bar $=50 \mu \mathrm{m}$. 

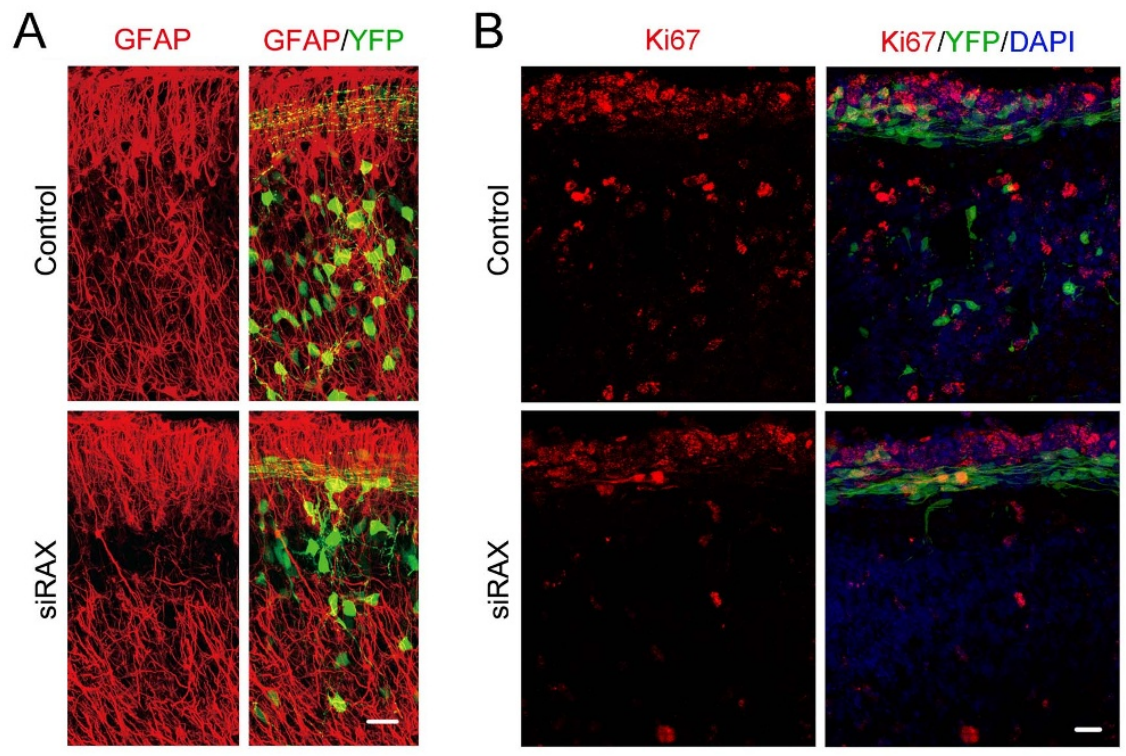

C

D
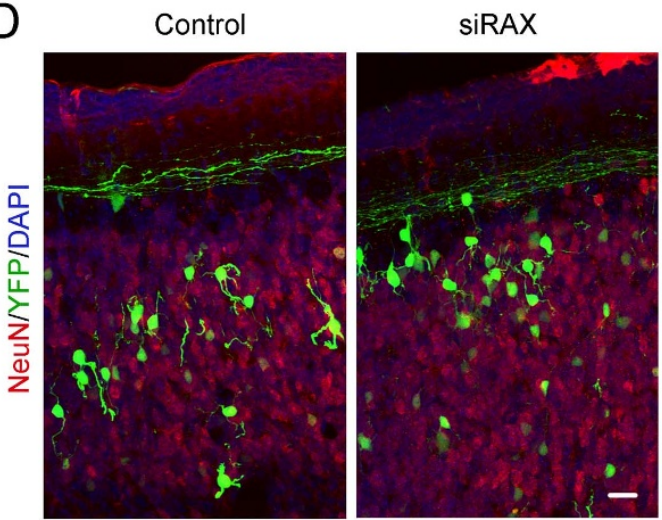

Figure 3 Effect of RAX knockdown on Bergman glia morphology and CGNs proliferation/differentiation. (A) Immunofluorescent images showing Bergman glia and their fibers which were visualized by GFAP staining. Scale bar $=25 \mu \mathrm{m}$. (B) At 48 hours post electroporation, sagittal sections of cerebella from both control and siRAX-treated groups were labeled with ki67 antibody (red) and DAPI (blue). Scale bar $=25 \mu \mathrm{m}$. (C) The percentage of ki67 positive cells in total transfected cells was calculated. (D) At 7 days post electroporation, coronal sections of cerebella from both control and siRAXtreated groups were labeled with NeuN antibody (red) and DAPI (blue). Scale bar $=25 \mu \mathrm{m}$.

PD6 mice. These mice were transfected with control or siRNA for RAX on PD4. We demonstrated that the migration of CGNs was significantly reduced in RAX knockdown group without apparent morphological alterations. The percentage of neurons that migrated within $100 \mu \mathrm{m}$ was significantly reduced in RAX knockdown group (Figures $4 \mathrm{~A}$ and B). Neurite growth was not affected by RAX knockdown (Figures $4 \mathrm{C}$ and D). These results supported that RAX played an important role in regulating the migration of CGNs.

The third motif of RAX is essential for its role in regulating CGN migration. RAX is a dsRNA binding protein and has three highly conserved motifs. We generated three recombinant RAX constructs. The first one was a wild type (WT) RAX with synonymous mutation in our siRNA targeting sequence to avoid silencing by the RAXsiRNA. The second one was truncated M1M2 (1-200 aa) including first two dsRBMs with the same mutation; the third one was truncated M3 (201-313 aa) including the third motif (Figure 5A). The expression of these constructs was detected by the expression of the Flag-tag (Figure 5B). When co-transfected with RAX siRNA, both the WT RAX and M3 constructs alleviated RAX knockdowninduced impairment of CGN migration in the developing cerebellum; however, the M1M2 construct failed to reverse the impairment of CGN migration (Figure 5C). These data indicated that the third motif of PACT/RAX was essential for regulating cell migration.

RAX regulation of CGN migration is PKR-independent. PACT/ RAX is the protein activator of PKR and the activation is mediated by the third motif of PACT/RAX ${ }^{9,22}$. We therefore sought to determine whether RAX regulation of neuronal migration depended on PKR. Up-regulation of PKR did not alleviate RAX knockdown-induced impairment of CGN migration in the developing cerebellum (Figures 6A and C). Furthermore, transfection of a dominantnegative PKR (K296R) construct did not aggravate the deficit in migration caused by RAX knockdown (Figures 6B and D). These results indicated that RAX regulation of CGN migration was independent of PKR.

PACT interacts with FAK and is involved in FAK phosphorylation in CGNs. FAK is an important regulator of cell migration ${ }^{18,19}$. In neurons, phosphorylation of FAK at serine 732 is critical for the organization of a small network of microtubules which is important for neural migration ${ }^{20}$. We found PACT interacted with FAK by GST pull-down assay (Figure 7A) which was also confirmed by coimmunoprecipitation (Figure 7B). In addition, RAX knockdown significantly decreased the phosphorylation of FAK at serine 732 in 
A
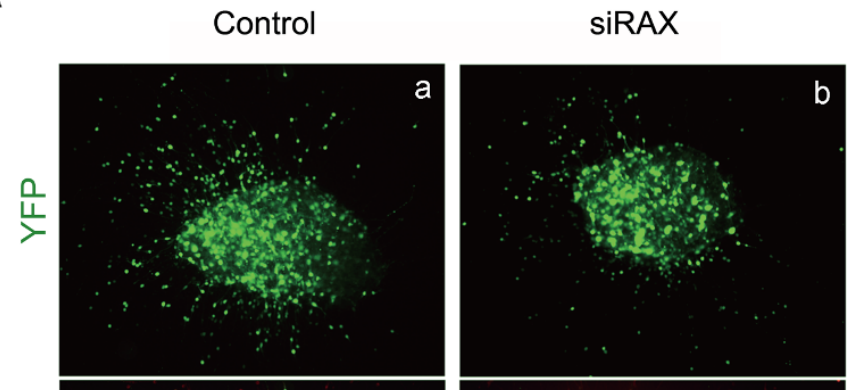

C
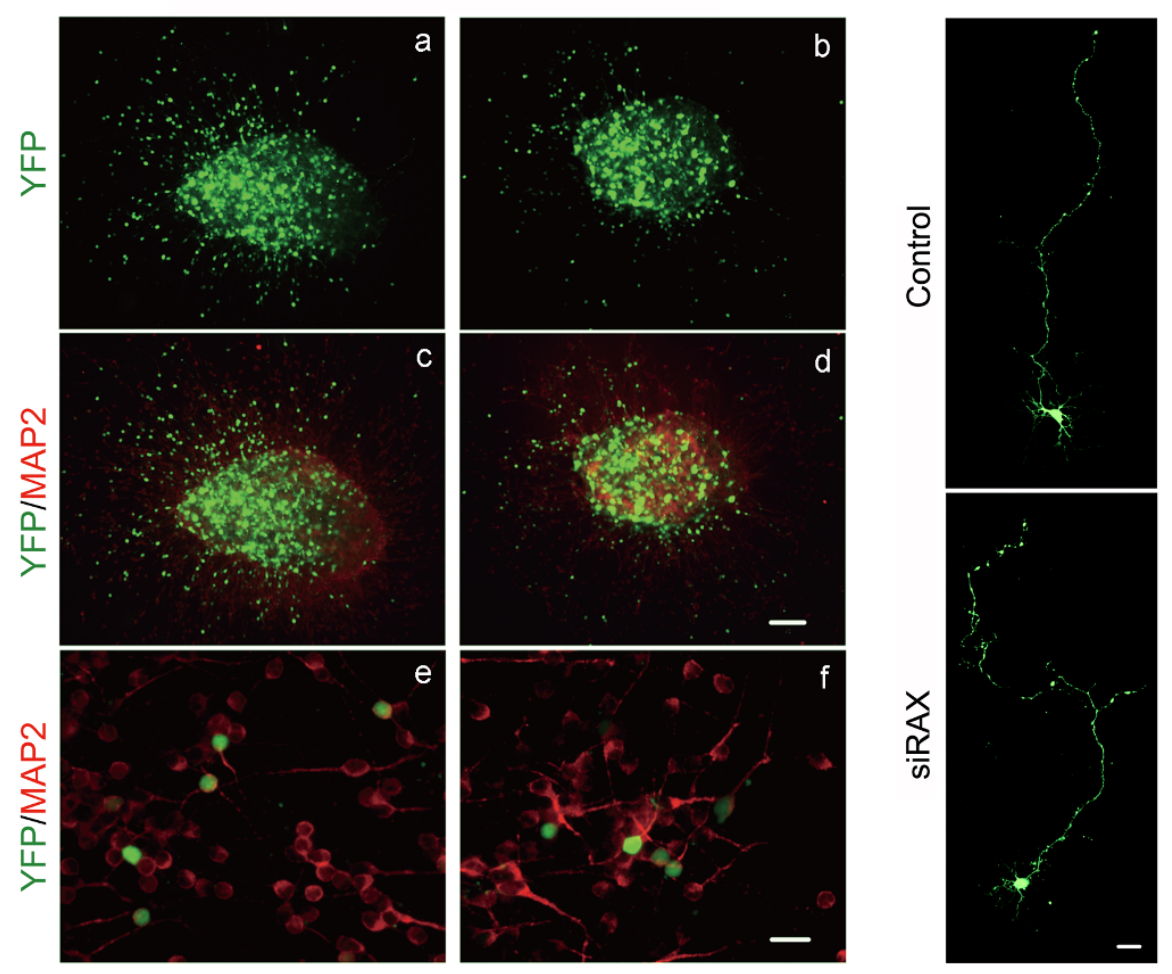

B

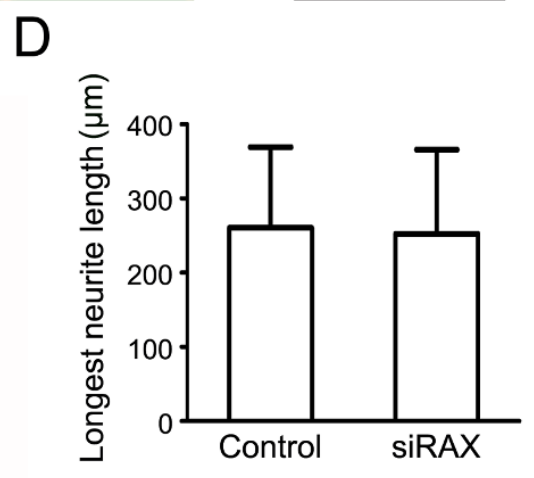

Figure $4 \mid$ Effect of RAX knockdown on CGN migration in cerebellar microexplants. (A) Cerebellar microexplants were prepared from PD6 mice that were transfected with siRNA for RAX and control siRNA by electroporation at PD4. MAP2 immunofluorescence (IF) was used to visualize neurons migrated out from microexplants after being cultured for 36 hours. YFP-positive signal indicated the transfected cells. Scale bars $=100 \mu \mathrm{m}(\mathrm{a}, \mathrm{b}, \mathrm{c}$ and $\mathrm{d})$ and $20 \mu \mathrm{m}$ (e and f). (B) The CGNs migrated out of microexplants were measured by the intensity of YFP fluorescence at various distances (0-100; 100-200 and 200-400 im), the intensity of YFP fluorescence within each area was counted and then expressed as a percentage of YFP intensity of the whole. Each data point is mean \pm s.d., $(\mathrm{n}=10),{ }^{*} \mathrm{p}<0.01$. (C) Images of higher magnification showing the migrated neurons. Scale bar $=20$ im. (D) The length of the longest neurite was quantified in 50 neurons. Each data point is mean \pm s.d. from three replicated experiments.

primary cultured CGNs (Figures 7C and D). Furthermore, RAX knockdown disrupted the organization of microtubules (Figure 7E); $58 \%$ of CGNs in the control group displayed prominent perinuclear microtubule cage while only $15 \%$ of CGNs transfected with RAX siRNA had this structure (Figure $7 F$ ).

\section{Discussion}

The human protein PACT and its mouse ortholog RAX were discovered independently as the cellular activator for the interferoninducible, dsRNA-dependent protein kinase $\mathrm{PKR}^{1,2}$. It has been reported that female flies homozygous for the dRax deletion are sterile $^{23}$, and display defects in CNS development ${ }^{17}$. The deletion of exon 8 of mouse Rax causes developmental defects, including reduced size and severe microtia ${ }^{14}$. The loss of the entire RAX gene in mice induced an early developmental lethality at a pre-implantation stage $^{17}$. These results indicate that RAX plays a role in the embryogenesis and development. In this study, we demonstrate that RAX plays an important role in the development of mouse cerebellum.

High levels of RAX in mouse cerebellum at PD4 and PD9 are consistent with the time period in which CGNs migrate from the EGL to the IGL. Immunohistochemical staining shows that RAX is highly expressed in EGL and Purkinje cells layer, suggesting a role in the migration of CGNs (Figure 1). We demonstrate that RAX knockdown causes granule cells to accumulate at the inner border of the EGL where the granule cell migration turns from tangential to radial, and RAX knockdown also affects the distance which granule cells migrate within the IGL (Figure 2). However, RAX knockdown does not damage the migration scaffold, the radial fibers of Bergmann glia; it does not affect either the proliferation/survival or differentiation of CGNs (Figure 3). It is therefore likely RAX regulates the ability of 
A

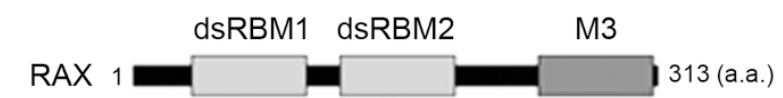

B

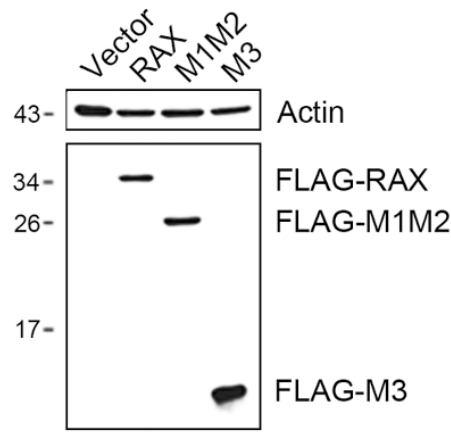

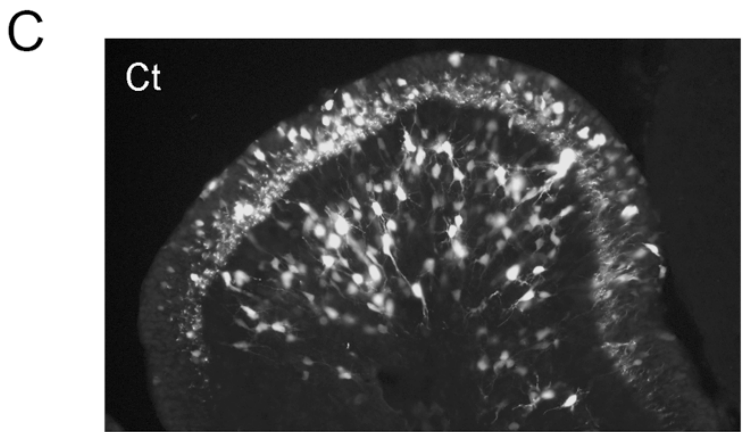
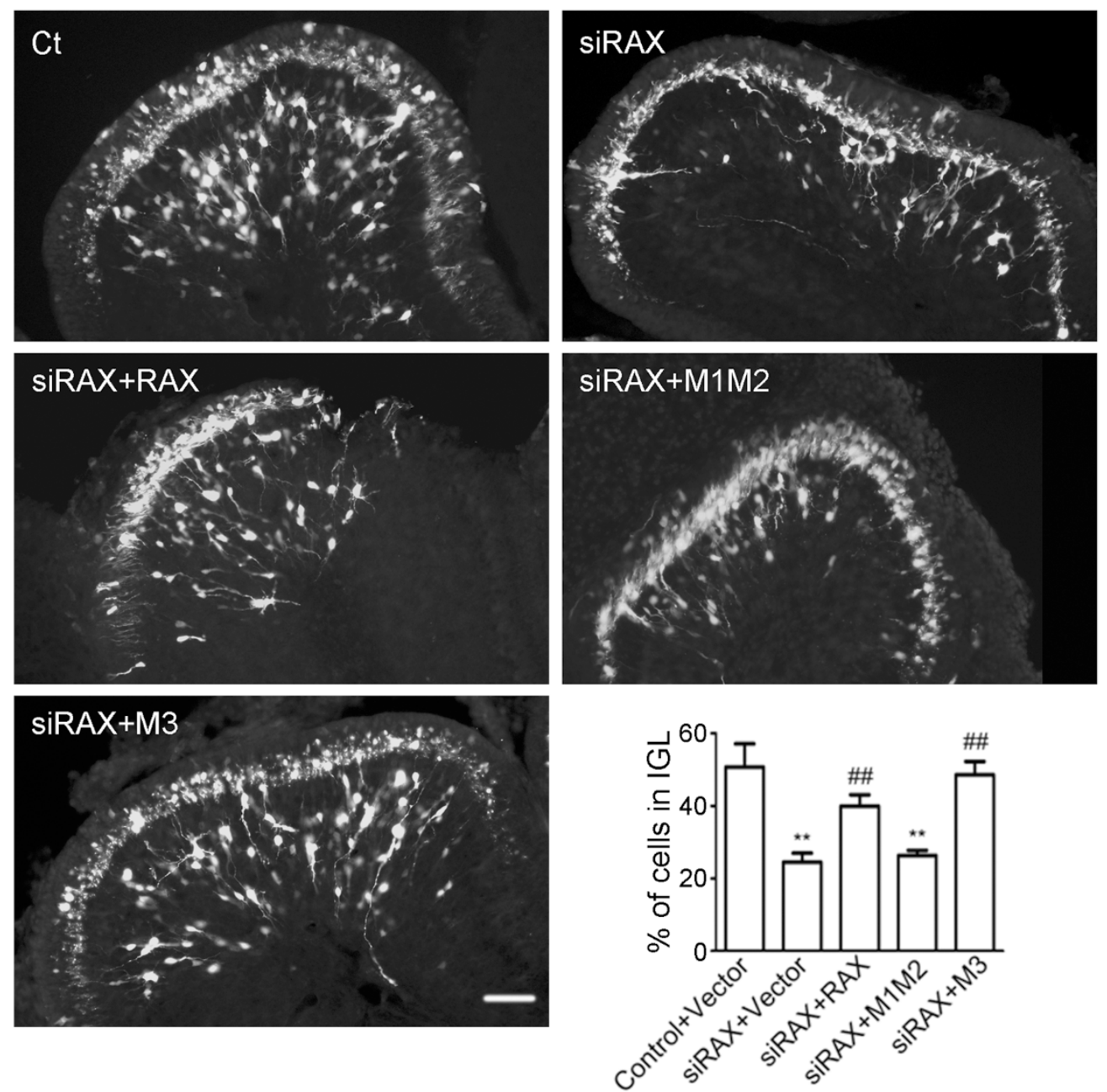

Figure 5 The third motif of RAX was required for the migration of CGNs in the developing cerebellum. (A) Schematic illustration of the wild type and truncated constructs (RAX, M1M2 and M3) of RAX. M1M2 construct contains two dsRBMs. M3 contains the third conserved motif. (B) The expression of truncated RAX in COS7 cells was measured by immunoblotting targeting Flag-tag. The cropped lines are used and full-length immunoblots are shown in Supplementary Information section (Supplementary Figure 3C). (C) Mouse pups were transfected with indicated RAX constructs (RAX, M1M2 and M3) or empty vectors (Vector) and siRAX by electroporation at PD4. Pups were sacrificed 72 hours after the electroporation, and cerebellar sagittal sections were prepared for the examination of cell migration. Scale bar $=50 \mu \mathrm{m}$. The percentage of cells that migrated into the IGL was calculated at 72 hours post electroporation. Each data point is mean \pm s.d. $(\mathrm{n}=5) .{ }^{* *} \mathrm{p}<0.01$, compared with Control + Vector group, \#\# $\mathrm{p}<0.01$, compared with siRAX + Vector group.

neuronal migration. This conclusion is supported by an in vitro study showing that RAX knockdown blocks neuronal migration in cultured cerebellar microexplants (Figure 4).

A recent study shows that $\mathrm{PKR}$ is involved in the migration of breast cancer cells; the activation of PKR suppresses cell motility by regulating the $\mathrm{p} 38 \mathrm{MAPK} / \mathrm{MK} 2 / \mathrm{LIMK} /$ cofilin pathway $^{24}$. Our results indicate that up-regulation of PKR expression could not rescue RAX knockdown-induced inhibition of CGN migration and co-expression of dominant negative PKR (K296R) does not aggravate the migration defect caused by RAX knockdown (Figure 6). Therefore, PACT/RAX regulation of CGN migration is likely independent of PKR.

PACT/RAX contains three independent motifs. The first two resemble dsRBM; the third one consisting of 66 residues appears to 
A
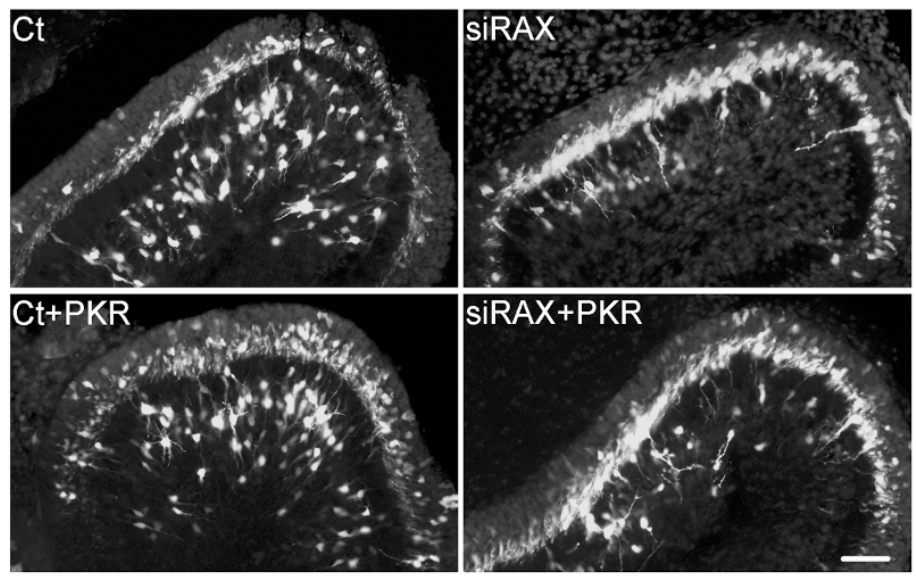

B
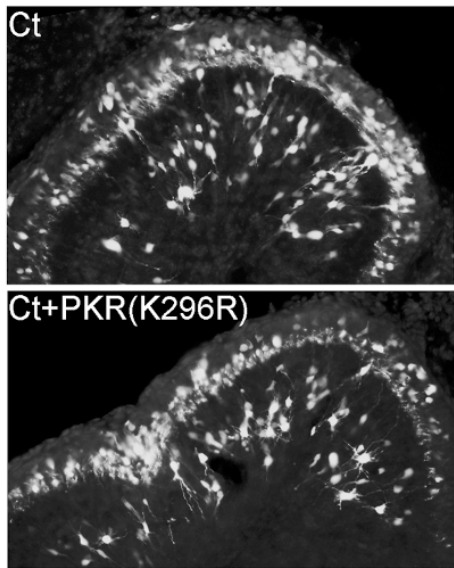

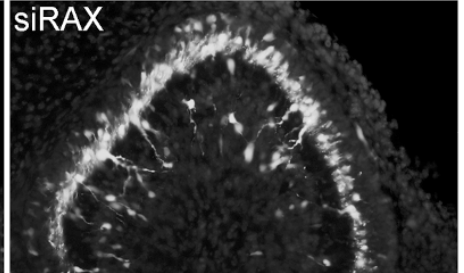

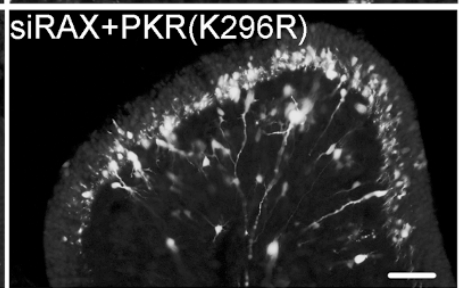

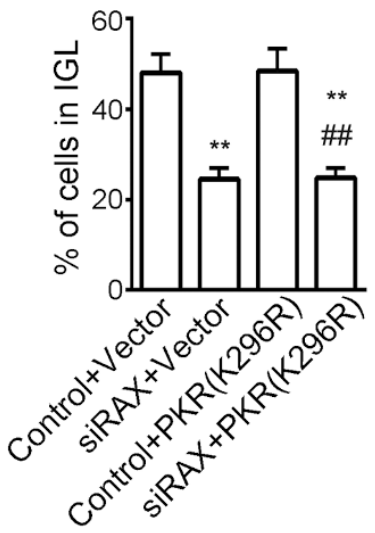

Figure 6 RAX knockdown inhibited CGN migration independent of PKR in vivo. (A) and (B) Mouse pups were co-transfected for indicated constructs [siRAX, PKR, PKR (K296R)] by electroporation at PD4, and sacrificed 72 hours after the electroporation. The cerebellar sagittal sections were prepared. YFP positive cells indicated transfected cells. Scale bar $=50 \mu \mathrm{m}$. (C) The percentage of YFP-positive granule cells migrated into the IGL (A) was calculated at 72 hours after the electroporation. Each data point is mean \pm s.d. $(\mathrm{n}=5) . * * \mathrm{p}<0.01$, compared with Control + Vector group, \#\# $\mathrm{p}<0.01$, compared with Control + PKR group. (D) The percentage of YFP-positive granule cells migrated into the IGL (B) was calculated at 72 hours after the electroporation. Each data point is mean \pm s.d. $(\mathrm{n}=5)$. ** $\mathrm{p}<0.01$, compared with Control + Vector group, \#\# $\mathrm{p}<0.01$, compared with Control + PKR(K296R) group.

be an activator for interacting proteins ${ }^{22}$. The dsRBMs of PACT (motif 1 and 2) interact with the dsRBMs in PKR; but the ability to activate PKR is imparted by the third motif of PACT that binds to the PKR kinase domain ${ }^{9}$. Binding of motif 3 to PKR causes PKR autophosphorylation by converting PKR from inactive conformation to an active one ${ }^{25}$. Although PACT's third motif binds weakly to PKR, it is necessary and sufficient to activate $\mathrm{PKR}^{9}$. Motif 1 and 2, on the other hand, may facilitate the process by mediating strong interaction between PACT and PKR. In mammalian cells, PACT itself is phosphorylated during various chemical stresses or growth factor withdrawal, and the phosphorylation may induce conformational changes of PACT to facilitate its third motif to associate with $\mathrm{PKR}^{8,26,27}$. We demonstrate that the expression of recombinant third motif of RAX is sufficient to reverse the defects in migration caused by RAX knockdown in the developing cerebellum. On the other hand, the expression of other two motifs fails to rescue CGNs from RAX knockdown-induced inhibition of neuronal migration (Figure 5). These results indicate that the third motif of PACT/RAX is critical for its regulation of neuronal migration. Interestingly, it appears that PKR is not involved in this process. Over expression of WT PKR does not alleviate the deficiency of CGN migration caused by RAX knockdown; dominant negative PKR (K296R PKR) is unable to aggravate the deficiency (Figure 6). PKR is not the only protein that interacts with the 3rd domain of PACT/RAX. Other well-known dsRBPs, such as Dicer and trans-activation responsive RNA-binding protein (TRBP), also interact with this domain. There may be other unknown factors that could interact with this domain. Currently, it is unclear how the third motif regulates CGN migration and what its interacting proteins are.

FAK plays an essential role in the regulation of cell migration ${ }^{18,19}$. By co-IP and GST pull-down assays, we demonstrate the interaction between PACT/RAX and FAK. The phosphorylation of FAK at serine 732 is critical for organization of a small network of microtubules that partially encompass the nucleus which is important for neuronal migration $^{20,28}$. We show that knockdown of PACT/RAX disturbs phosphorylation of FAK (S732) in CGNs (Figure 7). This suggests that PACT/RAX is upstream of FAK which is a critical mediator of neuronal migration.

Hence, our study unravels RAX is a new regulator of CGN migration during cerebellum development. Deficiency of RAX induces CGN migration defects, which is PKR independent, but the third motif of RAX is functionally necessary. Furthermore, knockdown of RAX decreases FAK serine 732 phosphorylation and disrupts the organization of microtubules. This study provides a novel insight 
A

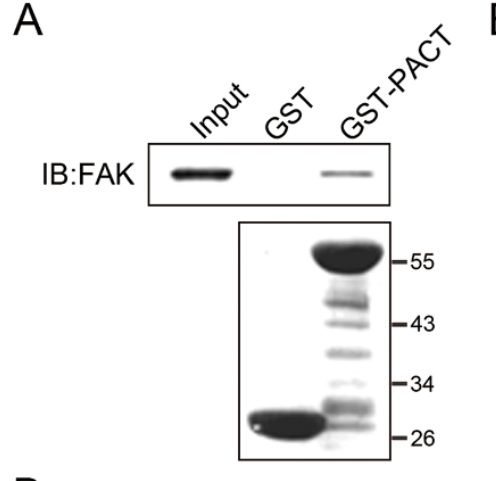

B

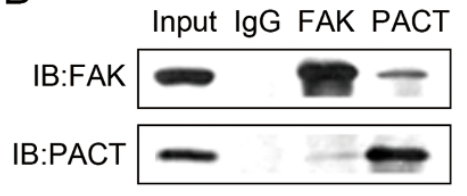

$E$
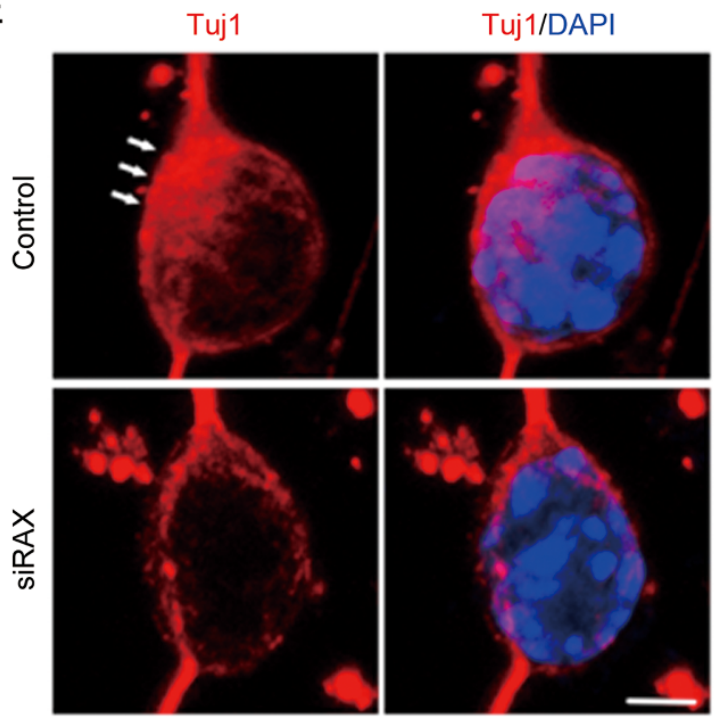

$\mathrm{F}$

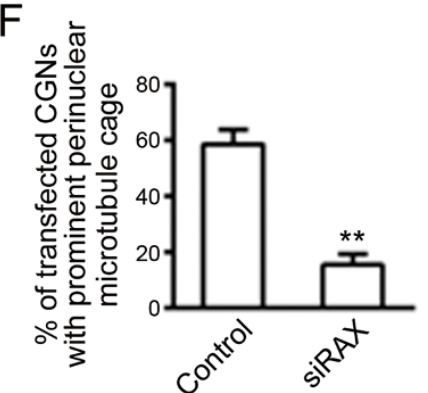

C

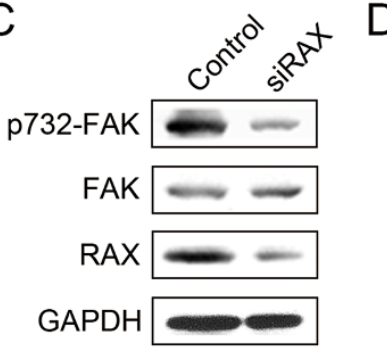

D

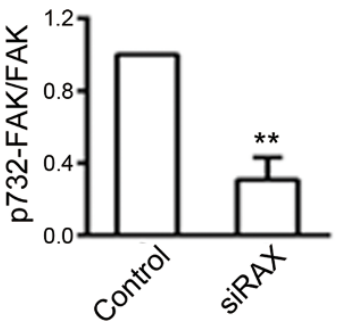


TCCCCCCGGGCTACTTATCGTCGTCATCCTTGTAATCGTGGTTCTCTGGAGAAATATTACTAAAC-3'; M3: FP: 5'-CCGCTCGAGATGATTTCTCTAACGAACGTGGTTGG-3' ${ }^{\prime}$, RP: 5' $^{\prime}$-TCCCCCCGGGCTACTTATCGTCGTCATCCTTGTAATCCTTTCTTTCTGCTATTATCTTTAAATAC-3'.

Human recombinant GST-PACT fusion protein was cloned into p-GEX-6p-2 vector using the primers as follows: FP 5'-CGGGATCCATGTCCCATAGCAGGCATCG-3', RP 5' -CCGCTCGAGCTACTTTCTTTCTGCTATTATCTTTAAATACT- $3^{\prime}$.

Human wild type PKR and dominant negative PKR (K296R) constructs were a kind gift from Dr. Alan Hinnebusch (National Institutes of Health, Bethesda, Maryland). All plasmids were verified by DNA sequencing.

In vivo gene delivery and quantification of CGN migration. Plasmids were delivered to the cerebellum by in vivo electroporation as described previously with some modifications ${ }^{29,30}$. Briefly, C57BL/6J mouse pups of postnatal day (PD) 4 were anesthetized, and the interparieta bone was exposed and pierced. Plasmids in sterile water containing $0.01 \%$ fast green was injected onto the surface of the cerebellum cortex. For each pup, $1.5 \mu \mathrm{l}$ of plasmid $(3 \mu \mathrm{g} / \mu \mathrm{l})$ was injected. Electroporation $(4$ pulses of $100 \mathrm{~V}$ for 50 microseconds with 950 microseconds intervals) was carried out using an Electro Square Porator (ECM 830, BTX). These pups were sacrificed 1, 2, 4 , or 7 days following electroporation and the cerebella were harvested.

Five slices with highest transfection efficiency from each mouse were selected for quantifying the migration of CGNs. The lobules $4 / 5$ or 6 were examined. We evaluated the percentage of cells that migrated into the IGL as well as the distance that they migrated inside the IGL (depth of migration). The depth of migration was determined by the distance the YFP-positive cell traveled within the IGL.

Culture and analysis of CGNs and cerebellar microexplants. Mouse pups that received in vivo gene deliveries on PD4 were sacrificed 48 hours following the electroporation, and the external granule layer (EGL) containing YFP positive cells was dissected under a dissecting microscope. Some tissues were prepared for primary CGN culture and others were used for cerebellar microexplant culture.

Primary CGN cultures were established as previously described ${ }^{31}$. Briefly, the dissected tissues were dissociated by trypsin incubation and trituration, and then centrifuged. The cell pellet was re-suspended in Neurobasal medium containing B27 (2\%), $\mathrm{KCl}(25 \mathrm{mM})$, glutamine $(1 \mathrm{mM})$, penicillin (100 units $/ \mathrm{ml})$ and streptomycin $(100 \mathrm{ug} / \mathrm{ml})$. Cells were plated onto poly-D-lysine $(50 \mu \mathrm{g} / \mathrm{ml})$-coated coverslips and maintained at $37^{\circ} \mathrm{C}$ in a humidified environment containing $5 \% \mathrm{CO}_{2}$ for 72 hours. The maximal neurite length in each YFP-positive CGN was measured with Image Pro plus software.

For cerebellar microexplant culture, the tissues were chopped to small pieces which were passed through a $308 \mu \mathrm{m}$ nylon mesh. The resulting tissue chunks were collected by sedimentation and resuspended in serum-free BME supplemented with hormones as previously described ${ }^{32}$. Cerebellar tissues were plated in coverslips that were double-coated with poly-D-lysine $(20 \mu \mathrm{g} / \mathrm{ml}$ in sterile water overnight at room temperature) and laminin ( $20 \mu \mathrm{g} / \mathrm{ml}$ in PBS for 3 hours at room temperature). After allowing for attachment for 1-2 hours, an appropriate volume of medium (DMEM/ F12 medium with $2 \mathrm{mM}$ glutamine, $0.1 \mathrm{mg} / \mathrm{ml} \mathrm{BSA}, 2.0 \% \mathrm{~N} 2,100 \mathrm{units} / \mathrm{ml}$ penicillin and $100 \mu \mathrm{g} / \mathrm{ml}$ streptomycin $)^{33}$ was added. Explants were cultured for 36 hours, and the rate of CGN migration was measured by the method described previously with some modifications ${ }^{34}$. Briefly, we delimited concentric areas of $0-100,100-200$ or $200-400 \mu \mathrm{m}$ width from the explant border. The intensity of YFP fluorescence in each area was measured using the Image-Pro Plus software and then expressed as a percentage of YFP intensity of the whole culture.

\section{Immunoprecipitation and immunobloting. Immunoprecipitation of FAK was} performed as previously described ${ }^{35}$. Briefly, an aliquot of cell lysate containing $200 \mu \mathrm{g}$ of proteins was pre-cleared with Protein A/G PLUS-Agarose. Protein A/G PLUS-Agarose was incubated with anti-FAK antibody for 2 hours at $4{ }^{\circ} \mathrm{C}$. Then the pre-cleared protein was treated with Protein A/G PLUS-Agarose/antibody complex at $4{ }^{\circ} \mathrm{C}$ overnight. Immunoprecipitates were collected by centrifugation at 3,000 g for 1 minute. After washing five times with 0.01 M PBS, the pellets were resuspended in $20 \mu \mathrm{l} 2 \times$ sodium dodecyl sulfate sample buffer. Then the protein sample was analyzed with SDS-PAGE and immunobolting as previously described ${ }^{31}$.

GST pull-down assay. Bacterial cells were lysed using the following buffer: $20 \mathrm{mmol} /$ L Tris-Cl, $2 \mathrm{mmol} / \mathrm{L}$ EDTA, $150 \mathrm{mmol} / \mathrm{L} \mathrm{NaCl}, 0.5 \% \mathrm{NP} 40$, pH 7.5.The bacterial lysate containing GST-PACT fusion protein was incubated with glutathioneSepharose $4 \mathrm{~B}$ beads at $4^{\circ} \mathrm{C}$ overnight. The beads were washed and then incubated with COS7 cell lysate for 6 hours. After washing, the bound proteins were denatured from the beads and subjected to SDS-PAGE ${ }^{36}$.

Immunohistochemistry. For immunohistochemical analysis, animals were anesthetized by intraperitoneal injection (IP) of chloral hydrate $(500 \mathrm{mg} / \mathrm{kg})$ and perfused with $10 \mathrm{ml}$ of saline, followed by $100 \mathrm{ml}$ of $4 \%$ paraformaldehyde in $0.1 \mathrm{M}$ phosphate buffer (PB, pH 7.2) ${ }^{37}$. Then brain tissues were removed and post-fixed in the same fixative overnight then transferred to $30 \%$ sucrose for an additional 24 hours and the cerebella were sectioned with a sliding microtome (Microm Laborgerate GmbH, Germany) at the thickness of $25 \mu \mathrm{m}$.

The procedure for immunohistochemistry has been previously described ${ }^{38}$. Briefly, cerebellum free-floating sections were incubated in $0.01 \mathrm{M} \mathrm{PBS}$ containing $3 \% \mathrm{H}_{2} \mathrm{O}_{2}$ and $40 \%$ methanol for 30 minutes at RT and then treated with $0.5 \%$ Triton X-100
(Sigma-Aldrich Co. LLC., St. Louis, MO) in PBS for 5 minutes. The sections were washed with $0.01 \mathrm{M}$ PBS three times and blocked with $1 \%$ BSA and $0.05 \%$ Triton X100 for 2 hours at RT and then incubated with primary antibody overnight at $4{ }^{\circ} \mathrm{C}$ followed by an incubation with secondary antibody for 2 hours at RT. After three washes in PBS, sections were incubated with Streptavidin, HRP-Conjugated (Calbiochem, Darmstadt, Germany) solutions for 2 hours and then developed in PBS containing $0.05 \% \mathrm{DAB}$ and $0.003 \% \mathrm{H}_{2} \mathrm{O}_{2}$.

Immunofluorescence. Fixed cerebellum sections, microexplants or cultured cells were treated with $0.5 \%$ Triton X-100 in PBS for 5 minutes, incubated with blocking solution (1\% BSA with $0.05 \%$ Triton X-100) for 30 minutes, and then treated with primary antibodies diluted in blocking solution for 1 hour. Cerebellum sections were blocked for 2 hours and then treated with primary antibodies diluted in blocking solution overnight. Sections were then washed with PBS and incubated with the secondary antibody and/or with Alexa Fluor ${ }^{\circledR} 555$ phalloidin for 1 hour. After three washes with PBS, the sections were stained with DAPI. The immunofluorescent images were captured under Zeiss LSM510 meta confocal microscope (Carl Zeiss MicroImaging Inc., Germany).

Statistical analysis. Statistical analyses were performed with PRISM software. Comparisons between two groups were evaluated by the Student's t test. Comparisons among multiple groups were performed with analysis of variance (ANOVA), followed by a two-tailed, unpaired Student's t test. Differences were considered significant when $\mathrm{p}<0.05$.

1. Ito, T., Yang, M. \& May, W. S. RAX, a cellular activator for double-stranded RNAdependent protein kinase during stress signaling. J Biol Chem 274, 15427-15432 (1999).

2. Patel, R. C. \& Sen, G. C. PACT, a protein activator of the interferon-induced protein kinase, PKR. EMBO J 17, 4379-4390 (1998).

3. Fierro-Monti, I. \& Mathews, M. B. Proteins binding to duplexed RNA: one motif, multiple functions. Trend Biochem Sci 25, 241-246 (2000).

4. Meurs, E. et al. Molecular cloning and characterization of the human doublestranded RNA-activated protein kinase induced by interferon. Cell 62, 379-390 (1990).

5. Koromilas, A. E., Roy, S., Barber, G. N., Katze, M. G. \& Sonenberg, N. Malignant transformation by a mutant of the IFN-inducible dsRNA-dependent protein kinase. Science 257, 1685-1689 (1992).

6. Samuel, C. E. The eIF-2 alpha protein kinases, regulators of translation in eukaryotes from yeasts to humans. J Biol Chem 268, 7603-7606 (1993).

7. Williams, B. R. PKR; a sentinel kinase for cellular stress. Oncogene 18, 6112-6120 (1999).

8. Chen, M., Chen, S. C. \& Pallen, C. J. Integrin-induced tyrosine phosphorylation of protein-tyrosine phosphatase-alpha is required for cytoskeletal reorganization and cell migration. J Biol Chem 281, 11972-11980 (2006).

9. Huang, X., Hutchins, B. \& Patel, R. C. The C-terminal, third conserved motif of the protein activator PACT plays an essential role in the activation of doublestranded-RNA-dependent protein kinase (PKR). Biochem J 366, 175-186 (2002).

10. Bennett, R. L. et al. RAX, the PKR activator, sensitizes cells to inflammatory cytokines, serum withdrawal, chemotherapy, and viral infection. Blood 108, 821-829 (2006).

11. Kok, K. H., Ng, M. H., Ching, Y. P. \& Jin, D. Y. Human TRBP and PACT directly interact with each other and associate with dicer to facilitate the production of small interfering RNA. J Biol Chem 282, 17649-17657 (2007).

12. Farazi, T. A., Juranek, S. A. \& Tuschl, T. The growing catalog of small RNAs and their association with distinct Argonaute/Piwi family members. Development 135, 1201-1214 (2008).

13. Lee, Y. et al. The role of PACT in the RNA silencing pathway. EMBO J 25, 522-532 (2006).

14. Rowe, T. M., Rizzi, M., Hirose, K., Peters, G. A. \& Sen, G. C. A role of the doublestranded RNA-binding protein PACT in mouse ear development and hearing. Proc Natl Acad Sci U S A 103, 5823-5828 (2006)

15. Peters, G. A., Seachrist, D. D., Keri, R. A. \& Sen, G. C. The double-stranded RNAbinding protein, $\mathrm{PACT}$, is required for postnatal anterior pituitary proliferation. Proc Natl Acad Sci U S A 106, 10696-10701 (2009).

16. Dickerman, B. K. et al. Missense mutation in the second RNA binding domain reveals a role for Prkra (PACT/RAX) during skull development. PloS one 6 , e28537 (2011).

17. Bennett, R. L. et al. RAX is required for fly neuronal development and mouse embryogenesis. Mech Dev 125, 777-785 (2008).

18. Hanks, S. K., Calalb, M. B., Harper, M. C. \& Patel, S. K. Focal adhesion proteintyrosine kinase phosphorylated in response to cell attachment to fibronectin. Proc Natl Acad Sci U S A 89, 8487-8491 (1992).

19. Parsons, J. T., Martin, K. H., Slack, J. K., Taylor, J. M. \& Weed, S. A. Focal adhesion kinase: a regulator of focal adhesion dynamics and cell movement. Oncogene 19, $5606-5613(2000)$.

20. Xie, Z., Sanada, K., Samuels, B. A., Shih, H. \& Tsai, L. H. Serine 732 phosphorylation of FAK by Cdk5 is important for microtubule organization, nuclear movement, and neuronal migration. Cell 114, 469-482 (2003). 
21. Watanabe, F. et al. Effects of FAK ablation on cerebellar foliation, Bergmann glia positioning and climbing fiber territory on Purkinje cells. Eur J Neurosci 27, 836-854 (2008).

22. Peters, G. A., Hartmann, R., Qin, J. \& Sen, G. C. Modular structure of PACT: distinct domains for binding and activating PKR. Mol Cell Biol 21, 1908-1920 (2001).

23. Forstemann, K. et al. Normal microRNA maturation and germ-line stem cell maintenance requires Loquacious, a double-stranded RNA-binding domain protein. PLoS Biol 3, e236 (2005).

24. Xu, M. et al. Double-stranded RNA-dependent protein kinase regulates the motility of breast cancer cells. PloS one 7, e47721 (2012).

25. Clemens, M. J. \& Elia, A. The double-stranded RNA-dependent protein kinase PKR: structure and function. J Interferon Cytokine Res 17, 503-524 (1997).

26. Wu, S. \& Kaufman, R. J. A model for the double-stranded RNA (dsRNA)dependent dimerization and activation of the dsRNA-activated protein kinase PKR. J Biol Chem 272, 1291-1296 (1997).

27. Bennett, R. L., Blalock, W. L. \& May, W. S. Serine 18 phosphorylation of RAX, the PKR activator, is required for PKR activation and consequent translation inhibition. J Biol Chem 279, 42687-42693 (2004).

28. Chacon, M. R. et al. Focal adhesion kinase regulates actin nucleation and neuronal filopodia formation during axonal growth. Development 139, 3200-3210 (2012).

29. Jia, Y., Zhou, J., Tai, Y. \& Wang, Y. TRPC channels promote cerebellar granule neuron survival. Nat Neurosci 10, 559-567 (2007).

30. Ohshima, T. et al. Cdk5 is required for multipolar-to-bipolar transition during radial neuronal migration and proper dendrite development of pyramidal neurons in the cerebral cortex. Development 134, 2273-2282 (2007).

31. Wang, X. et al. Thiamine deficiency induces endoplasmic reticulum stress in neurons. Neuroscience 144, 1045-1056 (2007).

32. Schilling, K., Dickinson, M. H., Connor, J. A. \& Morgan, J. I. Electrical activity in cerebellar cultures determines Purkinje cell dendritic growth patterns. Neuron 7 , 891-902 (1991).

33. Chou, D. K., Tobet, S. \& Jungalwala, F. B. Interaction of sulfoglucuronyl (HNK-1) carbohydrate and its binding protein, SBP-1, in microexplant cultures of rat cerebellum. J Neurosci Res 59, 188-201 (2000).

34. Kerjan, G. et al. The transmembrane semaphorin Sema6A controls cerebellar granule cell migration. Nat Neurosci 8, 1516-1524 (2005).

35. Wang, X., Fan, Z., Wang, B., Luo, J. \& Ke, Z. J. Activation of double-stranded RNA-activated protein kinase by mild impairment of oxidative metabolism in neurons. J Neurochem 103, 2380-2390 (2007).

36. Sun, H. et al. TRAF6 upregulates expression of HIF-1alpha and promotes tumor angiogenesis. Cancer Res 73, 4950-4959 (2013).
37. Ke, Z. J., DeGiorgio, L. A., Volpe, B. T. \& Gibson, G. E. Reversal of thiamine deficiency-induced neurodegeneration. J Neuropathol Exp Neurol 62, 195-207 (2003).

38. Yong, Y., Ding, H., Fan, Z., Luo, J. \& Ke, Z. J. Lithium fails to protect dopaminergic neurons in the 6-OHDA model of Parkinson's disease. Neurochem Res 36, 367-374 (2011).

\section{Acknowledgments}

We would like to thank Jacqueline A. Frank for reading this manuscript. We thank Dr. Alan Hinnebusch (National Institutes of Health) for providing human wild type PKR and dominant negative PKR (K296R) plasmids. This research was supported by grants from the Ministry of Science and Technology of China (2010CB912000), the National Natural Science Foundation of China (31271142), the Program of Clinical Research Center, Institute for Nutritional Sciences and Xuhui Central Hospital (CRC20100010). Dr. J. Luo was supported by a grant from NIH/NIAAA (AA015407).

\section{Author contributions}

Y.Y., Y.M., J.L. and Z.J.K. wrote the main manuscript text; Y.Y., Y.M., H.D., Z.F., Y.T. and C.Z. conducted experiments; Y.Y. and Y.M. prepared figures. All authors reviewed the manuscript

\section{Additional information}

Supplementary information accompanies this paper at http://www.nature.com/ scientificreports

Competing financial interests: The authors declare no competing financial interests.

How to cite this article: Yong, Y. et al. PACT/RAX Regulates the Migration of Cerebellar Granule Neurons in the Developing Cerebellum. Sci. Rep. 5, 7961; DOI:10.1038/srep07961 (2015)

(c) (i)

This work is licensed under a Creative Commons Attribution 4.0 International License. The images or other third party material in this article are included in the article's Creative Commons license, unless indicated otherwise in the credit line; if the material is not included under the Creative Commons license, users will need to obtain permission from the license holder in order to reproduce the material. To view a copy of this license, visit http://creativecommons.org/licenses/by/4.0/ 